\title{
Multivariate Statistical Analysis of Whole Brain Structural Networks Obtained Using Probabilistic Tractography
}

\author{
Emma C. Robinson ${ }^{1}$, Michel Valstar ${ }^{1}$, Alexander Hammers ${ }^{2}$, Anders Ericsson ${ }^{1}$, \\ A. David Edwards ${ }^{3}$, and Daniel Rueckert ${ }^{1}$ \\ ${ }^{1}$ Department of Computing, Imperial College, London. SW7 2BZ, UK \\ ${ }^{2}$ MRC Clinical Sciences Centre and Division of Neuroscience, Faculty of Medicine, \\ Imperial College, London. W12 ONN, UK \\ ${ }^{3}$ Department of Paediatrics, Imperial College, London. W12 ONN, UK
}

\begin{abstract}
This paper presents a new framework for the analysis of anatomical connectivity derived from diffusion tensor MRI. The framework has been applied to estimate whole brain structural networks using diffusion data from 174 adult subjects. In the proposed approach, each brain is first segmented into 83 anatomical regions via label propagation of multiple atlases and subsequent decision fusion. For each pair of anatomical regions the probability of connection and its strength is then estimated using a modified version of probabilistic tractography. The resulting brain networks have been classified according to age and gender using non-linear support vector machines with GentleBoost feature extraction. Classification performance was tested using a leave-one-out approach and the mean accuracy obtained was $85.4 \%$.
\end{abstract}

\section{Introduction}

Key to better understanding of brain function is a mapping of its underlying anatomical connectivity. Diffusion tensor MRI offers the possibility for approximation of this underlying neural microstructure through estimation of the diffusive profile of water molecules within brain tissue; known to be anisotropic or directed along white matter bundles. Connections between brain regions can then be estimated using tractography. In their simplest form tractography algorithms break down in areas of low diffusion anisotropy making them unsuited to studies of whole brain connectivity. In contrast, probabilistic tractography methods such as 1 have been shown to accurately represent thalamo-cortical grey matter connectivity in a wide variety of subject groups [2] [3]. In a natural progression of this approach we therefore seek to extend use of this technique to generate an approximation of the coordinated network of these connections in the whole brain.

Previous attempts at modelling functional and structural brain connections exist in [4] 5] 6] 15]. All of these studies have used graph theory and in particular the properties of small world graphs to characterise brain networks. Unfortunately, while this is useful for making generalised statements about the nature

D. Metaxas et al. (Eds.): MICCAI 2008, Part I, LNCS 5241, pp. 486-493, 2008.

(C) Springer-Verlag Berlin Heidelberg 2008 
and development of brain networks, it offers no quantifiable means of comparing the brain networks of different subjects. In this study we explore the potential for characterising brain networks of different subject groups using state-of-theart classification techniques from pattern recognition; performing classification through a combination of non linear support vector machines (SVM) [7] with Gentle Boosting 8 .

This paper makes two contributions: first, we present a model for the estimation of structural brain networks in adults which includes the calculation of parameters determining the probability as well as the strength of connections. Secondly we show how this model can be applied to a group of adult subjects on which classification is performed. Our results show that the brains of different subject groups can be distinguished by nature of their patterns of neural connectivity alone.

\section{Methods}

\subsection{Probabilistic Tractography and Connection Probability Estimation}

Traditional streamline tractography algorithms work by following the direction of maximum diffusion at each voxel, estimated from the principal eigenvector of the diffusion tensor. Unfortunately, this maximum likelihood approach offers no measurement of the confidence in the trajectory of the fibre tract. Thus, these approaches have problems tracking into areas of low diffusion anisotropy. Probabilistic schemes on the other hand, allow for tracking into areas of low certainty by directly estimating confidence in the model using, for example, Bayesian inference [1]. This results in a range of possible principal diffusion directions that can be sampled from during repeated streamlining resulting in a series of possible end points for each tract. Thus, given two regions of interest (ROIs) $A$ and $B$, the probability of $B$ being connected to the seed region $A$ (given the data, $Y$ ) can be calculated as the proportion of the total number of streamlines which reach region $B$ :

$$
P(\exists A \rightarrow B \mid Y)=\frac{n_{A \rightarrow B}}{n_{A}} .
$$

Here $n_{A \rightarrow B}$ denotes the number of streamlines seeded in region $A$ which reach region $B$ and $n_{A}$ denotes the number of streamlines seeded in region $A$.

In most cases the manually defined seed and target regions will however be very large in comparison to the tract volumes and thus division by entire seed volumes leads to an under-representation of the true likelihood of connection. Probability is therefore better defined in terms of the sub-region $s \in A$, whose voxels have a non-zero probability of seeding streamlines to $B$ :

$$
P(\exists s \rightarrow B \mid Y)=\frac{n_{A \rightarrow B}}{n_{s}} .
$$

Here $n_{s}$ denotes the number of streamlines seeded in sub-region $s$. 


\subsection{Connectivity Strength Estimation}

Whilst the probabilistic measures defined above give us some idea of the strength of belief that a connection exists, it does not measure the strength of the connection, i.e. the tract size. Influenced by confounding factors such as measurement noise and model inaccuracies, variations of the connection probability between subjects become difficult to interpret. Therefore we define another measure for the estimation of connection strength based on the concept of information flow and adapted from [6]. In this approach, local connection weights between voxels are determined by estimation of the diffusive transfer between them using integration of the orientation distribution function (ODF) over the solid angle $\beta$ $\left(=\frac{4 \pi}{26}\right)$ where the ODF radially projects a Gaussian probability density estimation $(P(\boldsymbol{R}))$ of the diffusion displacement $(R)$ along each of the 26 unit vectors $(\hat{u})$ joining each voxel $\left(\boldsymbol{r}_{\boldsymbol{i}}\right)$ with its neighbours:

$$
\begin{aligned}
& \mathrm{ODF}=\psi(\hat{u})=\int_{0}^{+\mathrm{inf}} R^{2} P(\boldsymbol{R}) d R \\
& P(\boldsymbol{R})=(4 \pi t)^{-\frac{3}{2}}(|D|)^{-\frac{1}{2}} \exp \frac{-\boldsymbol{R}^{T D^{-1} R}}{4 t} .
\end{aligned}
$$

Here $D$ represent the diffusion tensor and $\boldsymbol{R}=\hat{u} R$ is the projection of relative spin displacement of the water molecules along the unit vector $\hat{u}$.

By integrating over the solid angle $\beta$ (which describes the volume proportion over the unit sphere) it is possible to approximate the diffusion proportion $\left(P_{\text {diff }}(\hat{u})\right.$, proportionality constant $\left.C\right)$ calculated along each of the 26 projected directions in turn:

$$
P_{\text {diff }}(\hat{u})=\frac{1}{C} \int_{\beta} \psi(\hat{u}) d S .
$$

Local weights between voxels sampled during probabilistic tractography are determined by averaging this diffusion proportion in both directions along the unit arc joining voxels. Path or connection weights $(\zeta)$ between regions are determined by the mean of all $P_{\text {diff }}(\hat{u})$ obtained at each step of the probabilistic tracking. Finally, (anatomical) connection strengths (ACS) are determined by multiplication of $\zeta$ by an approximation of tract cross section taken from the mean number of voxels $\left(\boldsymbol{r}_{\boldsymbol{i}}\right)$ in the seed and target volumes $\left(V_{\text {seed }}, V_{\text {target }}\right)$ :

$$
\mathrm{ACS}=\frac{\zeta}{2}\left(\sum_{\forall \boldsymbol{r}_{i} \in V_{\text {seed }}} \boldsymbol{r}_{\boldsymbol{i}}+\sum_{\forall \boldsymbol{r}_{i} \in V_{\text {target }}} \boldsymbol{r}_{\boldsymbol{i}}\right)
$$

\subsection{Experiment: Extraction of Structural Brain Networks}

ROIs for seeding tractography were obtained by segmentation of the brain into 83 regions by label propagation [9] from multiple, manually-generated brain atlases. The resulting segmentations were then estimated using decision fusion as described in 10. As neurological connections typically start and end in the grey matter, cortical white matter was removed by multiplication of the anatomical 


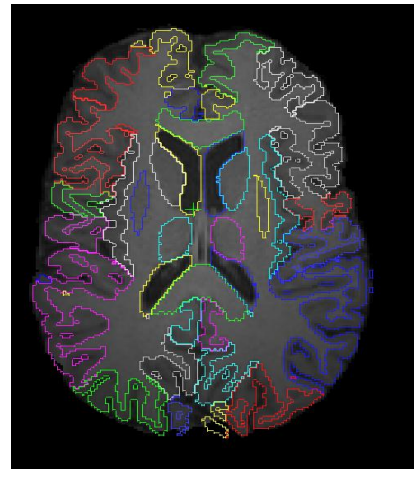

a)

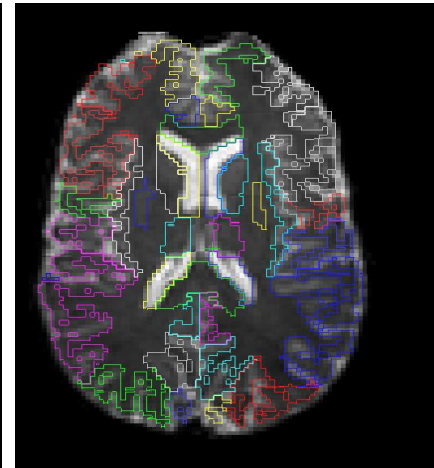

b)

Fig. 1. a) Structural (T1) segmentation and b) diffusion space segmentation after multiplication of anatomical segmentations with tissue segmentations to remove cortical white matter. Registration to diffusion space was performed by affine registration in two stages via T2 space.

segmentation with a tissue segmentation obtained using SPM5 [1]. Segmentations were transformed to the diffusion space (where tractography is traditionally performed) using affine registrations via intermediary T2 space (Fig. 1).

Next tractography was performed between pairs of regions in turn with only direct connections between regions being retained and connectivity being determined both probabilistically (2) and in terms of the connection strength (ACS) previously defined (6).

Results were represented as indices in $83 \times 83$ connection matrices (Fig. 2). Self-connections along the diagonal were removed and symmetry was enforced (since DTI is incapable of distinguishing between afferent and efferent biological connections). Connection vectors were formed by concatenating rows of each connectivity matrix. Finally, due to symmetry reasons, connections below the diagonal were removed as was the quadrant representing left-to-right connectivity which should be empty (assuming that all connections between brain lobes pass through and thus terminate at the corpus callosum).

\subsection{Statistical Analysis of Structural Brain Networks}

Gentle Boosting for Feature Extraction. Boosting is a technique which greatly improves the performance of classifiers by sequentially re-training weak classifiers on weighted data. It is used here solely for the purpose of feature extraction [8] choosing essentially orthogonal features on which to perform training using SVM. Over several boosting rounds weighted-least-squares regression was performed for every parameter. The best performing parameters were then selected as features and training samples were re-weighed according to their performance at each stage. 

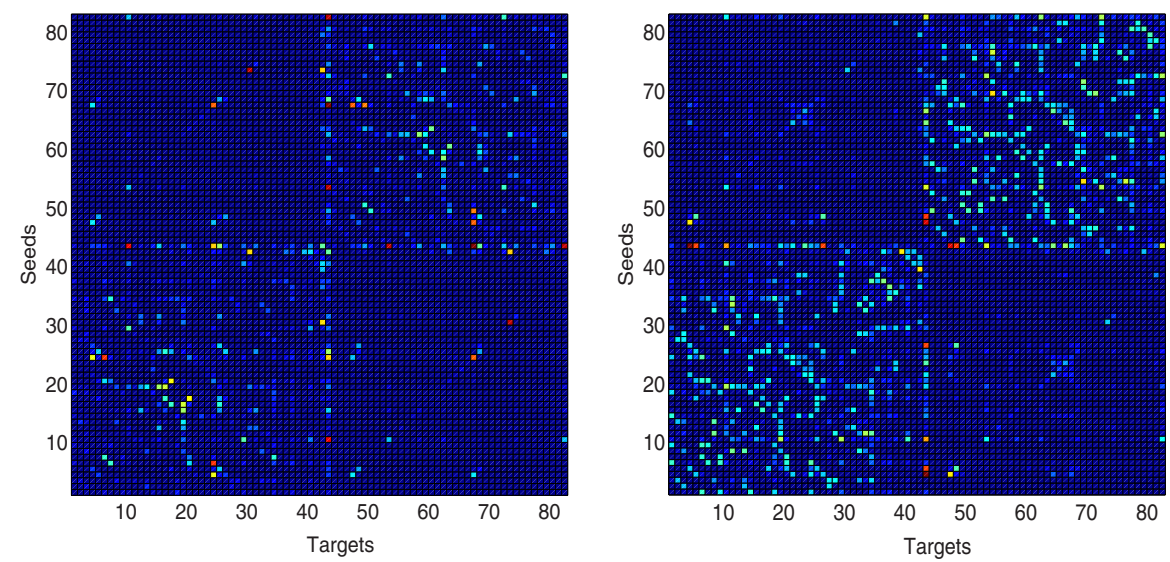

Fig. 2. Figure 1 Matrices representing mean ACS (left) and connection probability (right) for the network of connections between the 83 regions defined during anatomical segmentation

Classification using Support Vector Machines. Classification was performed using binary non-linear support vector machines [7.

In this technique, $\mathrm{n}$-dimensional data $\mathbf{x}_{\mathbf{i}}, i=1,2 \ldots n$; where $n$, in this case, is the number of GentleBoost features; is mapped through $\phi$ to a higher dimensional space $H\left(\phi: \boldsymbol{R}^{n} \mapsto H\right)$, in the hope that the data is linearly separable in that space. Separation is achieved by maximising the distance between the two parallel hyperplanes which form the boundaries between each class $y_{i} \in\{+1,-1\}$. Training points which define these hyperplanes are called the support vectors $\mathbf{s}$ (total number $N_{s}$ ).

Optimal separation is found by formulating the problem as a Lagrangian with multipliers $\alpha_{i}$. This has the added benefit of representing the problem in inner product form allowing for the higher dimensional problem to be solved in a lower dimensional space using a Mercer kernel [8] : $K\left(\mathbf{x}_{\mathbf{i}}, \mathbf{x}_{\mathbf{j}}\right)=\left\langle\phi\left(\mathbf{x}_{\mathbf{i}}\right), \phi\left(\mathbf{x}_{\mathbf{j}}\right)\right\rangle$. After training the decision function on a test point $\mathbf{x}$ is then given by:

$$
f(x)=\sum_{i=1}^{N_{s}} \alpha_{i} y_{i} K\left(\mathbf{s}_{\mathbf{i}}, \mathbf{x}\right)=b
$$

Where, b refers to the bias of the hyperplanes from the origin. A Gaussian radial basis function kernel was used: $K\left(\mathbf{x}_{\mathbf{i}}, \mathbf{x}_{\mathbf{j}}\right)=e^{-\|\boldsymbol{X}-\boldsymbol{Y}\|^{2} / 2 \sigma_{2}}$

Leave one out tests. Classification was performed $\mathrm{N}$ times each with $\mathrm{N}-1$ image vectors in the training set. The remaining image vector was then used for testing.

Performance was calculated using the $F_{\text {measure, }}$, the harmonic mean of classification precision and recall, where precision $(P)$ is a measure of the number of true positives (those correctly labelled as belonging to a class) over the total 
(true positives plus false positives) and, recall $(R)$ is a measure of true positives divided by the number of objects that should have been labelled in that class (true positives plus false negatives): $F_{\text {measure }}=\frac{2 \cdot P \cdot R}{P+R}$

\section{Results}

Connectivity analysis was performed on 174 adult brains (89 female); median age 45 years (range: 20-86 years). Scanning was performed on a Philips 3 Tesla system. T1 and T2 images were acquired prior to diffusion weighted imaging using 3D MRPRAGE and dual echo weighted imaging. Single shot echo planar DTI was acquired in 15 non collinear directions using the following parameters: TR $12000 \mathrm{~ms}$, TE $51 \mathrm{~ms}$, slice thickness $2 \mathrm{~mm}$, voxel size $=1.75 \times 1.75 \times 2 \mathrm{~mm}^{3}$, $\mathrm{b}$ value $1000 \mathrm{~s} / \mathrm{mm}^{2}$.

Connectivity results are shown in Fig. 2 Rows and columns have been permuted such that left and right brain regions are separated and corpus callosum runs through the middle. Left-right quadrants are predominantly empty (as expected) except for connections across the mid-line caused by registration error (Fig. 1). In addition results for connection strength and probability show significantly different intensity patterns reflecting the differences in interpretation between a high probability that a connection exists and inference of a strong connection.

Leave one out results for classification according to age (group $1=20$-49 years; group $2=50-86$ years) or gender are shown in Table 1 , In both cases the classifier performed better on the results for connectivity strength. Discriminating features (connections) identified by the classifiers are shown in Tables 2 and 3 . For men and women these predominantly include connections to and from the corpus callosum, temporal gyri and areas of the limbic system known to differ between

Table 1. $F_{\text {measure }}$ results of the classification

\begin{tabular}{rc}
\hline & Age Gender \\
\hline Strength & $87.9 \% 86.8 \%$ \\
Probability & $85.1 \% 81.6 \%$ \\
\hline
\end{tabular}

Table 2. Discriminating features defining classification for connection strength: $\mathrm{P}=$ posterior; $\mathrm{A}=$ anterior; $\mathrm{S}=$ superior; $\mathrm{I}=$ inferior; $\mathrm{L}=$ left; $\mathrm{R}=$ right. Derivation described in 2.4

\begin{tabular}{rc}
\hline \multicolumn{1}{c}{ Age } & Gender \\
\hline Postcentral to A Temporal (L) & Presubgenual Frontal to Cingulate (A) (L) \\
Subgenual Frontal to I. Frontal (L \& R) & Corpus to Cingulate (P) (L) \\
Straight Gyrus to Medial Temporal (R) & Corpus to S. Temporal (A) (L) \\
Nucleus Accumbens to I. Frontal (R) & Putamen to Pallidum (L) \\
\hline
\end{tabular}


Table 3. Discriminating features defining classification for connection probability: $\mathrm{P}=$ posterior; $\mathrm{A}=$ anterior; $\mathrm{S}=$ superior; $\mathrm{I}=$ inferior; $\mathrm{L}=$ left; $\mathrm{R}=$ right. See 2.4 .

\begin{tabular}{cc}
\hline Age & Gender \\
\hline Putamen to Cingulate (P) (L and R) & Presubgenual Frontal to Cingulate (P) (L) \\
Brainstem to Cingulate (P) (L) & Insula to Lingual (L) \\
Brainstem to S. Frontal (L) & Thalamus to P. Orbital (L) \\
S.Temporal (P) to Cingulate (P) (R) & Thalamus to Putamen (L) \\
\hline
\end{tabular}

genders. For age, differences predominantly include features of the frontal lobe and its associated connections.

\section{Discussion}

Fundamental to understanding differences in brain function between the sexes or over time is identification of differences in the underlying connectivity which monitors behaviour. Studies into structural brain connectivity across subject groups thus far have been limited mostly to major white matter structures. These have pointed to an overall degeneration in fractional anisotropy (FA) and therefore connection strength with age [12] and regional differences in FA in the corpus callosum between men and women 13 . However this is the first study that has pointed to global changes in connectivity across subject groups.

Whilst it is possible that ACS; calculated from seed and target volumes; may be sensitive to changes in brain size and therefore interpretation of any features should be approached with caution, there are similarities here with reports from functional studies which suggest that female brains exhibit higher bilateral connectivity 14] (reflected by the significance of connections to the corpus callosum) as well as a prominence of age-discriminating features connecting the pre-frontal lobes, whose white matter integrity is known to correlate with changes in behavioural performance over time 12 .

It is true that more sophisticated diffusion models such as diffusion spectrum imaging have produced more detailed connected networks [15] and therefore we acknowledge that steps do need to be taken to improve accuracy of the model through better registration (so that ROIs can be assumed correct with a high degree of accuracy) and extension to multiple-fibre tractography models (such that all connections are well approximated). Nevertheless, notwithstanding any limitations, this group study has shown great potential for the discrimination of subject groups by nature of their whole brain connectivity. Studies of this kind have the potential to highlight the key connective features which best describe anatomical segregation across subject groups. And, though care must be taken to verify the results with histology and current clinical opinion, if studied in line with functional research such features may help to vastly improve understanding of the origins of behavioural change. Furthermore, extensions to multiple class or unsupervised learning approaches may allow us to model healthy ageing. 


\section{References}

1. Behrens, T., Woolrich, M., Jenkinson, M., Johansen-Burg, H., Nunes, R., Clare, S., Matthews, P., Brady, J., Smith, S.: Characterization and propagation of uncertainty in diffusion-weighted mr imaging. Magn. Res. Med. Anal. 50, 1077-1088 (2003)

2. Behrens, T., Johansen-Burg, H.: Relating connectional architecture to grey matter function using diffusion imaging. Phil. Trans. R. Soc. B 360, 903-911 (2005)

3. Counsell, S., Dyet, L., Larkman, D., Nunes, R., Boardman, J., Allsop, J., Fitzpatrick, J., Srinivasan, L., Cowan, F., Hajnal, J., Rutherford, M.: Edwards: Thalamo-cortical connectivity in children born preterm mapped using probabilistic magnetic resonance tractography. Neuroimage 34, 896-904 (2006)

4. Honey, C., Kötter, R., Breakspear, M., Sporns, O.: Network structure of cerebral cortex shapes functional connectivity on multiple time scales. PNAS 24, 1024010245 (2007)

5. Achard, S., Salvador, R., Whitcher, B., Suckling, J., Bullmore, E.: A resilient low frequency small-world human brain functional network with highly connected association cortical hubs. J. Neuroscience 26, 63-72 (2006)

6. Iturria-Medina, Y., Canales-Rodrígues, E., Melie-García, L., Valdés-Hernández, P., Martínez-Montes, E., Alemán-Gómez, Y., Sánchez-Bornot, J.: Characterizing brain anatomical connections using diffusion weighted MRI and graph theory. Neuroimage 36, 645-660 (2007)

7. Burges, C.: A tutorial on support vector machines for pattern recognition. Data mining and knowledge discovery 2, 121-167 (1998)

8. Valstar, M.F., Pantic, M.: Fully automatic facial action unit detection and temporal analysis. In: CVPR, pp. 149-126 (2006)

9. Heckemann, R., Hajnal, J., Aljabar, P., Rueckert, D., Hammers, A.: Automatic anatomical brain MRI segmentation combining label propagation and decision fusion. Neuroimage 33, 115-126 (2006)

10. Aljabar, P., Heckemann, Hammers, A., Hajnal, J.V., Rueckert, D.: Classifier selection strategies for label fusion using large scale atlas databases. In: Medical Image Computing and Computer-Assisted Intervention, pp. 523-531 (2006)

11. Ashburner, J., Friston, K.: Unified segmentation. Neuroimage 26, 839-851 (2005)

12. Madden, D., Whiting, W., Huettel, S., White, L., MacFall, J., Provenzale, J.: Diffusion tensor imaging of adult age differences in cerebral white matter:relation to response time. NeuroImage 21, 1174-1181 (2004)

13. Oh, J., Song, I., Lee, J., Kang, H., Park, K., Kang, E., Loo, D.: Tractography guided statistics in diffusion tensor imaging for the detection of gender difference in fiber integrity of the corpora callosa. NeuroImage 36, 606-616 (2007)

14. Davatzikos, C., Resnick, S.: Sex differences in anatomic measures of interhemispheric connectivity; correlations with cognition in women but not men. Cerebral Cortex 8, 635-640 (1998)

15. Hagmann, P., Kurant, M., Gigandet, X., Thiran, P., Wedeen, V., Meuli, R., Thiran, J.: Mapping human whole-brain structural networks with diffusion MRI. PLoSONE 7, 597 (2007) 